\title{
Sudden Cardiac Death Caused by a Septic Coronary Artery Embolism as the First Clinical Presentation of Infective Endocarditis
}

Eojin $\mathrm{Kim}^{1}$, Taehwa Baek ${ }^{2}$ Sookyung Lee ${ }^{3}$, Han Na Kim ${ }^{4}$

${ }^{1}$ Department of Pathology, Samsung Medical Center, Sungkyunkwan University School of Medicine, Seoul, Korea, ${ }^{2}$ Hyundai Forensic Medical Service, Seoul, Korea, ${ }^{3}$ Division of Forensic Medical Examination, National Forensic Service, Wonju, Korea, ${ }^{4}$ Division of Forensic Investigation, National Forensic Service, Wonju, Korea

Received: July 15, 2021 Revised: November 11, 2021 Accepted: November 17, 2021

\section{Correspondence to} Han Na Kim Division of Forensic Investigation, National Forensic Service, 10 Ipchun-ro, Wonju 26460, Korea Tel: +82-33-902-5220

Fax: +82-33-902-5911

E-mail:pavane07@gmail.com
This report describes an uncommon and fatal case of myocardial infarction due to coronary embolus arising from vegetation in the aortic valve with a background of infective endocarditis (IE). There are various causes of fatal IE. Myocardial infarction due to septic emboli is rare. We report a case of sudden death in a 69-year-old woman with hyperlipidemia and no known cardiac disease. She had severe general weakness and was hospitalized for colonoscopy. The patient unexpectedly presented with cardiac arrest and died. The autopsy showed total occlusion of the left anterior descending artery by an embolus, which originated from the septic vegetation of the aortic valve. Myocardial infarction from septic emboli associated with IE can be fatal and manifested as the first presentation. In autopsy practice of deceased patients with IE, careful examination of the coronary arteries is required.

Key Words: Autopsy; Infective endocarditis; Vegetation; Myocardial infarction

\section{Introduction}

Patients with infective endocarditis (IE) exhibit variable complications, such as congestive heart disease, periannular complications, acute renal failure, abscesses, and systemic embolic events [1,2]. Myocardial infarction caused by an embolism of vegetation is a rare complication of IE. Coronary embolisms may present with ischemic chest pain, arrhythmia, valvular defects, and pulmonary edema. However, the first and only symptom of myocardial infarction could be sudden death in approximately $25 \%-40 \%$ of all patients with myocardial infarction [3]. Moreover, it is a lethal complication that leads to death in more than half of IE patients [4]. Here, we report an autopsy case of an unusual death due to myocardial infarction associated with IE. 


\section{Case Report}

A 69-year-old woman with no known cardiac disease complained of general weakness. She was maintained on atorvastatin, a hyperlipidemia medication. She planned to undergo colonoscopic polypectomy for a known colonic polyp. However, colonoscopy was postponed because of severe general weakness. The patient was hospitalized for further assessment. On examination, there were no signs or symptoms that could be clinically suspected of a specific disease. After routine blood tests and intravenous fluid administration, the patient was discharged from the hospital. However, in the ward bathroom, she was found unconscious.
Even with prompt cardiopulmonary resuscitation, she was pronounced dead. Subsequently, a postmortem pathological examination was performed.

The gross findings of the heart were consistent with cardiomegaly, weighing $440 \mathrm{~g}$. Multiple, whitish, and friable vegetations were observed in all cusps of the aortic valve (Fig. 1A, B). Gross and subsequent histopathological examination of the myocardium revealed no definite lesions. Total occlusion of the left anterior descending artery was identified on the serial section of the coronary artery (Fig. 1C). The other coronary vessels were not obstructed and showed mild atherosclerotic changes. The aortic valve harbored fibrotic and calcific changes, which were identified

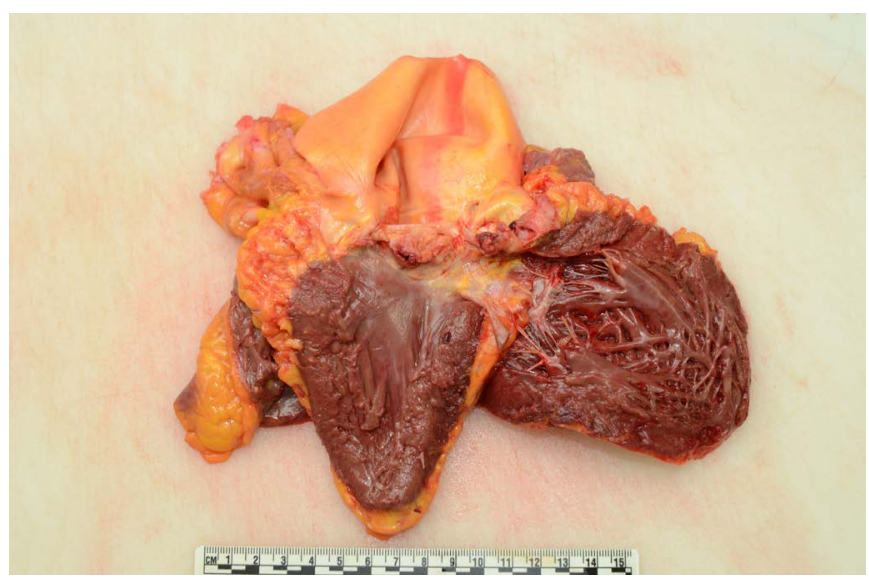

A

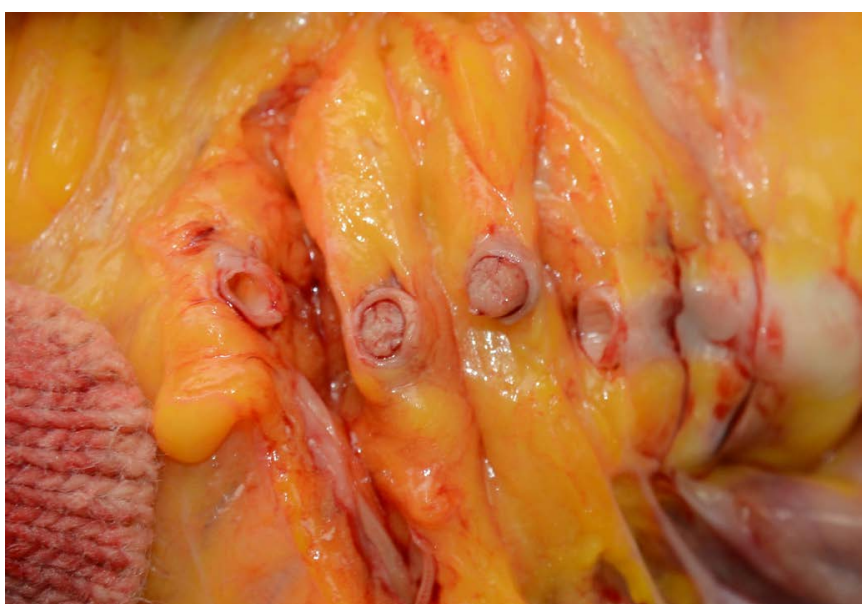

C

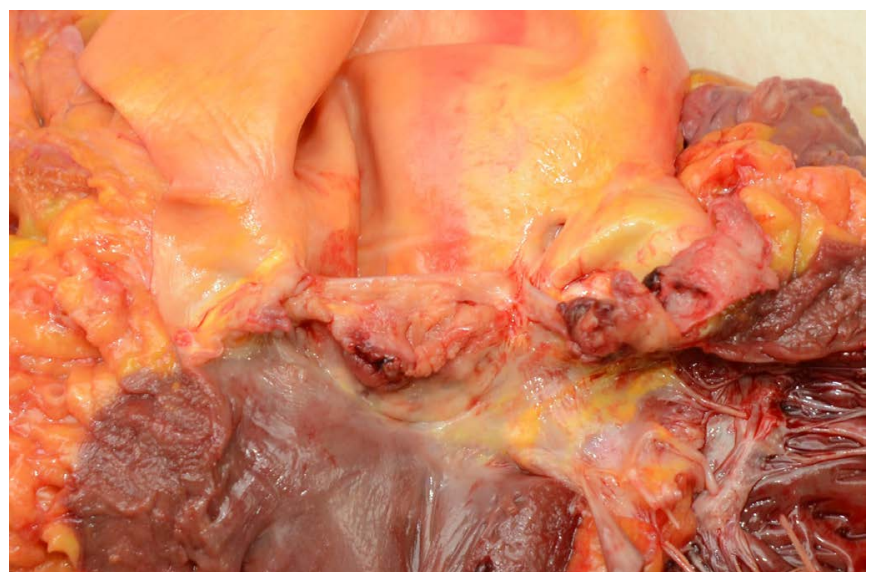

B

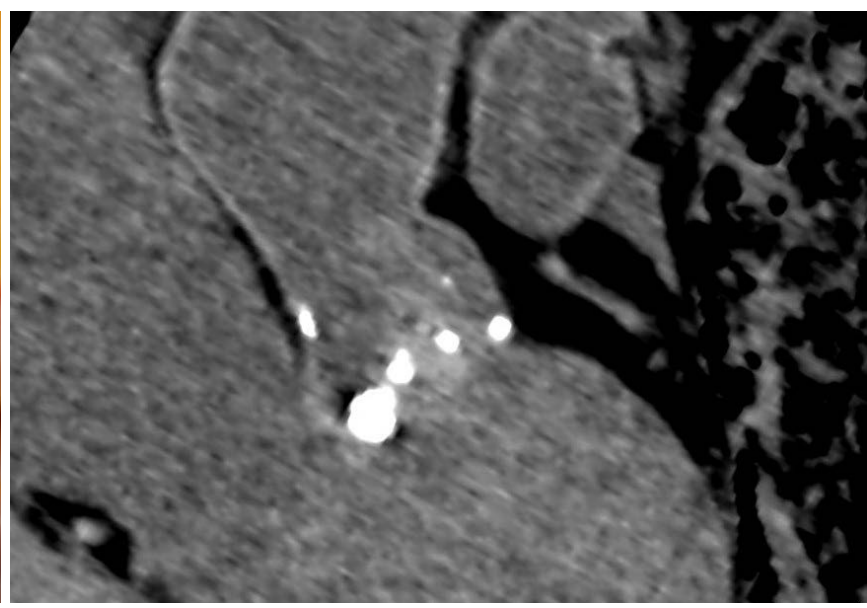

D

Fig. 1. Gross examination and computed tomography findings of the heart. (A, B) The heart showed cardiomegaly, and multiple friable vegetations were denoted on the aortic valve. (C) Serial sectioning $(5 \mathrm{~mm})$ of the left main coronary artery revealed a whitish embolus, occupying the total internal diameter of the coronary artery. (D) Postmortem computed tomography revealed a calcified aortic valve and illdefined and amorphous vegetation. 
using postmortem computed tomography (Fig. 1D).

Microscopic examination revealed multiple nodular calcified changes with perivalvular fibrosis and interstitial fibrosis of the cardiac muscles, which destroyed the valve structure (Fig. 2A). Inflammation was identified on the full thickness of the valve, which contained acute and chronic inflammatory cells and fibrosis (Fig. 2B). The vegetation on the aortic valve comprised nodular and calcified thrombotic debris with fibrin deposition, which included some macrophagecontaining bacterial colonies (Fig. 2C). The septic embolus occupying the coronary artery was also composed of thrombotic debris, calcification, and dead organisms, showing the same histopathologic findings as those of the vegetation of the aortic valve (Fig. 3A, B). Systemic embolisms at other sites were not detected on autopsy examination. Furthermore, other organs, such as those in the central nervous system, liver, kidney, and spleen, were also unremarkable.

\section{Discussion}

Myocardial ischemia associated with IE has been identified by several pathophysiological mechanisms, including extrinsic coronary compression of the ostium due to periannular complications (i.e., abscesses or pseudoaneurysms), large vegetations, severe aortic valve regurgitation, and coronary artery embolization $[4,5]$. A previous report identified the main mechanism of myocardial ischemia in a patient with IE involving coronary compression due to a large periannular aortic complication [4]. Coronary artery embolisms secondary

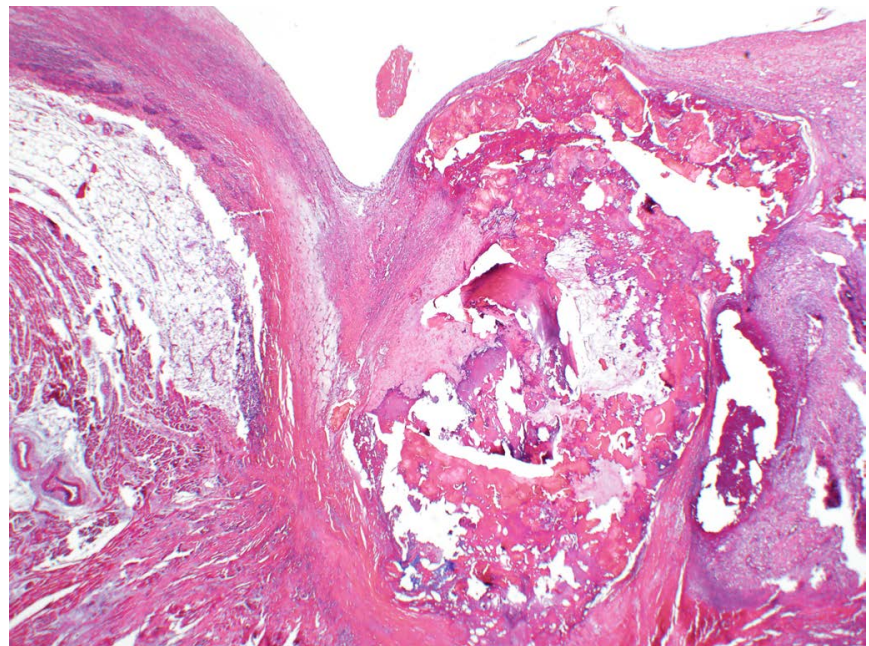

A

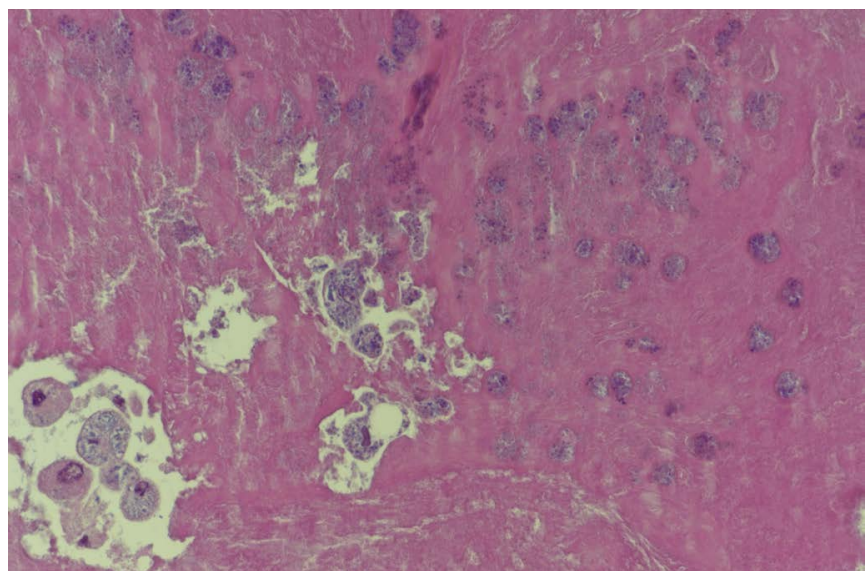

C

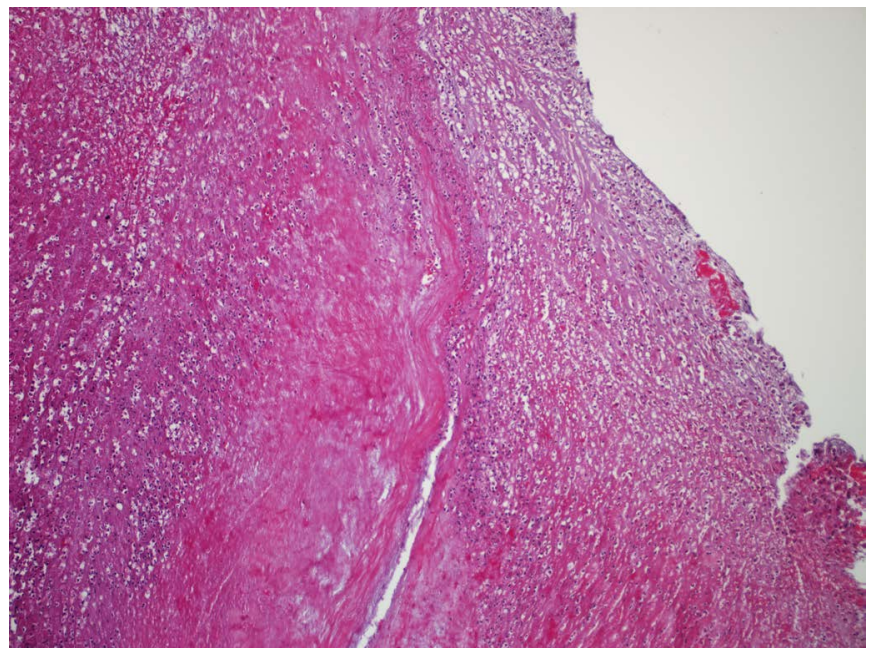

B

Fig. 2. Microscopic findings of the aortic valve with vegetation. (A) The calcified aortic valve and interstitial fibrosis of the perivalvular cardiac muscles were shown $(H \& E, \times 40)$. (B) The inflammation on the valve demonstrated infiltration of acute and chronic inflammatory cells and karyorrhexis of neutrophils $(H \& E, \times 100)$. (C) On higher magnification, the vegetation included fibrinoid material and some colonies of bacteria in the portion that contained several macrophages $(H \& E$, $\times 400)$. 


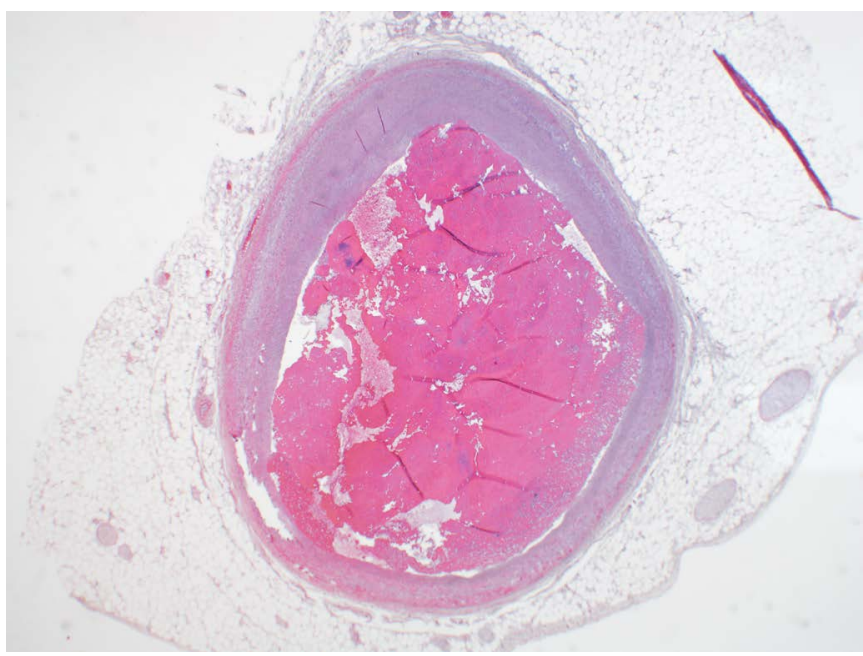

A

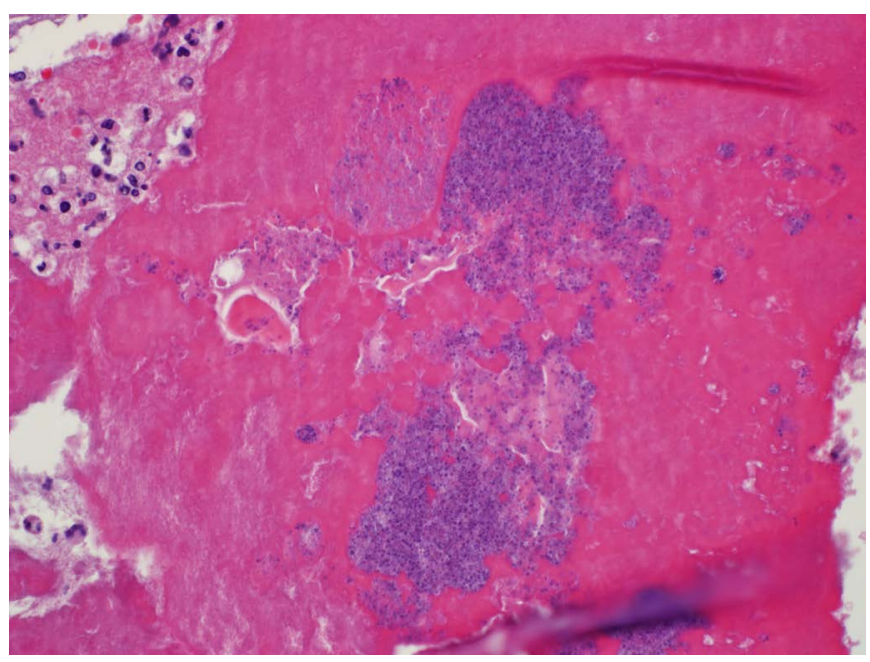

B

Fig. 3. Microscopic findings from the coronary artery (left anterior descending artery). (A) The coronary artery showed total occlusion by septic emboli $(H \& E, \times 12.5)$. (B) Fibrin deposition, inflammatory cells, and dead organisms were demonstrated in the embolic fragment $(H \& E, \times 400)$.

to IE are a rare cause of myocardial ischemia in patients with IE.

The main complications of IE are heart failure, systemic embolization, and uncontrolled infection. Systemic embolic events are a common complication of native valve IE, observed in $20 \%-50 \%$ of cases [6]. However, most cases of embolic events involve cerebral localization (>65\%) [1]. Stroke is a fatal complication of IE and is associated with higher morbidity and mortality rates [7]. Other frequently involved sites of embolisms are the kidneys (60\%) and spleen (44\%) [1]. Furthermore, more than half of the embolic events appear within a week before and after the initiation of antibiotic therapy [8]. Our postmortem study revealed acute coronary embolism as the first clinical presentation of IE in a patient who did not receive any therapy.

Acute coronary syndrome secondary to IE is infrequent, and its incidence is difficult to determine. After the case report by Virchow in 1856, several autopsies or cases in the clinical setting have been reported [9-11]. A large cohort revealed that 133 of 629 patients (21.1\%) developed a systemic embolic event; the coronary artery was involved in two patients (0.3\%) [8]. In another series of $104 \mathrm{IE}$ patients, 11 (10.6\%) showed myocardial infarction resulting from septic embolism [5]. Moreover, some authors have revealed the clinical significance of coronary embolism in patients with IE based on the fact that 14 of a total of 481 patients (2.9\%) with endocarditis presented with acute coronary syndrome, of which $64 \%$ died [4].

Thrombi on heart valves are referred to as vegetation. Vegetation on cardiac valves is a hallmark of IE. Microscopically, the vegetation contains fibrin, inflammatory cells, bacteria, and other organisms. After treatment with antibiotics, the bacterial colonies cannot be identified or a greater proportion of dead organisms is shown than in the viable condition [12]. Granulation tissues, fibrosis, chronic inflammatory cells, and calcification may occur over time.

The optimal therapy for myocardial infarction with IE is controversial. Antiplatelet treatment is not recommended in this setting because the risk of embolism is not reduced [13]. In addition, the risk of hemorrhagic complications with the use of anticoagulants is increased, leading to fatal outcomes [14]. The best strategy for reducing the risk of embolism is appropriate antibiotic therapy [15]. However, the risk of embolism is highest when antibiotic treatment is initiated within the first 2 weeks $[16,17]$. Therefore, an early surgical approach is recommended to confer the benefits of embolic event prevention given that the patient is devoid of an ongoing ischemic event or hemodynamic instability. The main indications of surgery are the size and mobility of the vegetation, history of embolisms, type of microorganisms, and 
duration of antimicrobial therapy [18]. Traditional percutaneous coronary intervention has questionable benefits for patients with myocardial infarction and IE. Ongoing acute ischemia and hemodynamic instability are the only indications for the procedure. Careful treatment decisions are necessary because of the variable results of the procedure $[19,20]$.

Coronary septic embolism is a very rare and a potentially fatal complication of IE that results in myocardial infarction. However, if the embolus is only locally present, it may be difficult to identify. Therefore, during autopsy of deceased patients with IE, careful examination of the coronary arteries is required.

ORCID: Eojin Kim: https://orcid.org/0000-0003-31116754; Taehwa Baek: https://orcid.org/0000-0001-6554076X; Sookyung Lee: https://orcid.org/0000-00028939-9526; Han Na Kim: https://orcid.org/0000-00019249-5466

\section{Conflicts of Interest}

No potential conflict of interest relevant to this article was reported.

\section{References}

1. Mocchegiani R, Nataloni M. Complications of infective endocarditis. Cardiovasc Hematol Disord Drug Targets 2009;9:240-8.

2. Na JY, Cho WY, Park JW, et al. Infective endocarditis: an autopsy case report with literature review. Korean J Leg Med 2014;38:7882.

3. Dolinak D, Matshes E, Lew EO. Forensic pathology: principles and practice. Amsterdam: Elsevier Academic Press; 2005. p. 72-6.

4. Manzano MC, Vilacosta I, San Roman JA, et al. Acute coronary syndrome in infective endocarditis. Rev Esp Cardiol 2007;60:2431.

5. Tiurin VP, Korneev NV. The mechanisms of the development and diagnosis of myocardial infarct in septic endocarditis. Ter Arkh 1992;64:55-8.

6. Habib G, Lancellotti P, Antunes MJ, et al. 2015 ESC Guidelines for the management of infective endocarditis: The Task Force for the
Management of Infective Endocarditis of the European Society of Cardiology (ESC). Endorsed by: European Association for CardioThoracic Surgery (EACTS), the European Association of Nuclear Medicine (EANM). Eur Heart J 2015;36:3075-128.

7. Thuny F, Avierinos JF, Tribouilloy C, et al. Impact of cerebrovascular complications on mortality and neurologic outcome during infective endocarditis: a prospective multicentre study. Eur Heart J 2007;28:1155-61.

8. Fabri J Jr, Issa VS, Pomerantzeff PM, et al. Time-related distribution, risk factors and prognostic influence of embolism in patients with left-sided infective endocarditis. Int J Cardiol 2006;110:334-9.

9. Brunson JG. Coronary embolism in bacterial endocarditis. Am J Pathol 1953;29:689-701.

10. Taherkhani M, Haybar $H$, Khaheshi I, et al. A rare case of acute myocardial infarction (AMI) due to infective endocarditis: clinical case presentation.J Indian Coll Cardiol 2015;5:160-3.

11. Castelli JB, Almeida G, Siciliano RF. Sudden death in infective endocarditis. Autops Case Rep 2016;6:17-22.

12. Burke A, Tavora F. Practical cardiovascular pathology. Philadelphia, PA: Lippincott Williams \& Wilkins; 2010. p. 349-63.

13. Chan KL, Dumesnil JG, Cujec B, et al. A randomized trial of aspirin on the risk of embolic events in patients with infective endocarditis. J Am Coll Cardiol 2003;42:775-80.

14. Hunter AJ, Girard DE. Thrombolytics in infectious endocarditis associated myocardial infarction.J Emerg Med 2001;21:401-6.

15. Chu VH, Sexton DJ, Cabell CH, et al. Repeat infective endocarditis: differentiating relapse from reinfection. Clin Infect Dis 2005;41:406-9.

16. Thuny F, Di Salvo G, Belliard O, et al. Risk of embolism and death in infective endocarditis: prognostic value of echocardiography: a prospective multicenter study. Circulation 2005;112:69-75.

17. Vilacosta I, Graupner C, San Roman JA, et al. Risk of embolization after institution of antibiotic therapy for infective endocarditis. J Am Coll Cardiol 2002;39:1489-95.

18. Thuny F, Beurtheret S, Mancini J, et al. The timing of surgery influences mortality and morbidity in adults with severe complicated infective endocarditis: a propensity analysis. Eur Heart J 2011;32:2027-33.

19. Glazier JJ. Interventional treatment of septic coronary embolism: sailing into uncharted and dangerous waters. J Interv Cardiol 2002;15:305-7.

20. Taniike M, Nishino M, Egami Y, et al. Acute myocardial infarction caused by a septic coronary embolism diagnosed and treated with a thrombectomy catheter. Heart 2005;91:e34. 